\section{Phylogeography of Toxoplasma gondii Points to a South American Origin}

Emilie Bertranpetit ${ }^{\mathrm{a}}$, Thibaut Jombart ${ }^{\mathrm{b}}$, Emmanuel Paradis ${ }^{\mathrm{c}}$, Hilda Pena ${ }^{\mathrm{d}}$, Jitender Dubey $^{\mathrm{e}}$, Chunlei Suf ${ }^{\mathrm{f}}$ Aurélien Mercier ${ }^{\mathrm{a}}$, Sébastien Devillard ${ }^{\mathrm{g}, 1,2}$, Daniel Ajzenberg ${ }^{\mathrm{h}, 1,2}$

aINSERM UMR_S 1094, Neuroépidémiologie Tropicale, Laboratoire de ParasitologieMycologie, Faculté de Médecine, Université de Limoges, Limoges, 87025, France ${ }^{b}$ MRC Centre for Outbreak Analysis and Modelling, Department of Infectious Disease Epidemiology, School of Public Health, Imperial College London, United Kingdom 'Institut des Sciences de l'Évolution, Université Montpellier/CNRS/IRD/EPHE, Place Eugène Bataillon - CC 065, 34095 Montpellier cédex 05, France ${ }^{d}$ Departamento de Medicina Veterinária Preventiva e Saúde Animal, Faculdade de Medicina Veterinária e Zootecnia, Universidade de São Paulo, São Paulo, Brazil e United States Department of Agriculture, Agricultural Research Service, Beltsville Agricultural Research Center, Animal Parasitic Diseases Laboratory, Building 1001, Beltsville, Maryland, 20705-2350, USA

${ }^{f}$ Department of Microbiology, University of Tennessee, Knoxville, Tennessee, 379960845, USA

gUniversité de Lyon, F-69000, Lyon ; Université Lyon 1 ; CNRS, UMR 5558, Laboratoire de Biométrie et Biologie Evolutive, F-69622, Villeurbanne, France ${ }^{h}$ Centre National de Référence (CNR) Toxoplasmose / Toxoplasma Biological Resource Center (BRC), Centre Hospitalier-Universitaire Dupuytren, Limoges, 87042, France and INSERM UMR_S 1094, Neuroépidémiologie Tropicale, Laboratoire de ParasitologieMycologie, Faculté de Médecine, Université de Limoges, Limoges, 87025, France

${ }^{1}$ Correspondence and requests for materials should be addressed to S.D (email: sebastien.devillard@univ-lyon1.fr Tel: +33472448111) and D.A. (email: aiz@unilim.fr Tel: +33555056160 )

${ }^{2}$ Authors contributed equally 


\section{1}

42

\section{Abstract}

Toxoplasma gondii, a protozoan found ubiquitously in mammals and birds, is the etiologic agent of toxoplasmosis, a disease causing substantial Public Health burden worldwide, including about 200,000 new cases of congenital toxoplasmosis each year. Clinical severity has been shown to vary across geographical regions, with South America exhibiting the highest burden. Unfortunately, the drivers of these heterogeneities are still poorly understood, and the geographical origin and historical spread of the pathogen worldwide are currently uncertain. A worldwide sample of $168 T$. gondii isolates gathered in 13 populations was sequenced for five fragments of genes (140 single nucleotide polymorphisms from 3,153 bp per isolate). Phylogeny based on Maximum likelihood methods with estimation of the time to the most recent common ancestor (TMRCA) and geostatistical analyses were performed for inferring the putative origin of $T$. gondii. We show that extant strains of the pathogen likely evolved from a South American ancestor, around 1.5 million years ago, and reconstruct the subsequent spread of the pathogen worldwide. This emergence is much more recent than the appearance of ancestral T. gondii, believed to have taken place about $11 \mathrm{My}$ ago, and follows the arrival of felids in this part of the world. We posit that an ancestral lineage of T. gondii likely arrived in South America with felids and that the evolution of oral infectivity through carnivorism and the radiation of felids in this region enabled a new strain to outcompete the ancestral lineage and undergo a pandemic radiation.

Keywords: Toxoplasma gondii, phylogeography, Maximum likelihood phylogeny, time to the most recent common ancestor (TMRCA), genetic diversity 


\section{Introduction}

Toxoplasmosis imposes a substantial disease burden across the world. Serological studies demonstrate its presence in virtually every country, with seroprevalence exceeding $60 \%$ in some parts of South America, Africa, and South-East Asia (Pappas et al., 2009). While asymptomatic in most patients, toxoplasmosis is a major cause of uveitis in immunocompetent patients and a potentially life-threatening illness in immunocompromised patients and fetuses (Montoya and Liesenfeld, 2004). Congenital toxoplasmosis alone has a yearly global incidence of about 200,000 cases, causing a burden exceeding 1 million disability-adjusted life year (DALYs) (Torgerson and Mastroiacovo, 2013). The overall disease burden attributable to all forms of toxoplasmosis is certainly far greater, and highest in South America where ocular toxoplasmosis is unusually frequent and severe (Glasner et al., 1992; de-la-Torre et al., 2008; Gilbert et al., 2008; Torgerson and Mastroiacovo, 2013).

The extent to which host parasite genetics, host immune status, and exposure rate contribute to the increased severity of toxoplasmosis in South America is unclear, but differences in the genetic makeup of $T$. gondii strains are likely to play a major role (Khan et al., 2006; Gilbert et al., 2008). In North America, Europe, Africa, and Asia, the population structure of $T$. gondii is dominated by a few prevalent clonal strains, whereas much greater genetic diversity is seen in tropical South America where the populations lack sign of recent genetic bottleneck and clonal structure seen in the other parts of the world (Shwab et al., 2014; Lorenzi et al., 2016). 
Unfortunately, the drivers of the pathogen's genetic diversity are still poorly understood, and the origin of extant lineages of $T$. gondii remains controversial. Recent work suggested a potential South American origin, while another study advocated that comigration with felids led to the divergence of South American strains from pre-existing North American ones (Lehmann et al., 2006; Khan et al., 2007). The estimation of the time to the most recent common ancestor (TMRCA) of extant lineages is also disputed, with estimates ranging from 150,000 to $10^{7}$ years (Morrison, 2005; Khan et al., 2007). In the present study, using a large collection of genetic sequences of $T$. gondii sampled worldwide, we reconstructed the phylogeography of $T$. gondii as a basis to address the controversial questions regarding the evolution of this parasite and its geographical origin.

\section{Materials and Methods}

\subsection{Collection of T. gondii strains and selection of markers}

A total of 168 T. gondii strains from 13 populations collected worldwide in North America, South America, the Caribbean, Europe, Asia, and Africa, were used in this study (Supplementary information and S1 Table). Our collection was specifically designed to ensure extensive geographic coverage of strains clustered in true populations, and includes a large number of strains from Africa and China, which were so far underrepresented or absent in the previous studies (Lehmann et al., 2006; Khan et al., 2007). A preliminary genetic analysis with 15 microsatellite markers (Ajzenberg et al., 2010) was performed to exclude clones of strains sampled in the same area. Each isolate was sequenced in both directions for five markers (GRA6, GRA7, SAG3, UPRT1 
and UPRT7) that had the highest polymorphic rates after a preliminary analysis of genetic polymorphism of 30 fragments of genes retrieved from GenBank and ToxoDB (Supplementary information and S2 Table).

\subsection{Phylogenetic analysis.}

Hammondia hammondi is the most closely parasite related to T. gondii and was used as an outgroup in phylogenetic analyses. Sequences of the $H$. hammondi strain H.H.34 corresponding to T. gondii GRA6, GRA7, SAG3, UPRT1 and UPRT7 sequences were retrieved from GenBank and ToxoDB, and aligned with MUSCLE (Edgar, 2004). We used ape and pegas $\mathrm{R}$ packages to extract haplotypes and build phylogenetic trees with three distance-based methods: NJ, BIONJ, and FastME which were used as starting trees for the $\mathrm{ML}$ analyses. Maximum likelihood phylogenetic analyses were performed with the R package phangorn using four partitions of the sequence data by crossing two criteria: exons vs. introns on one side, and GRA6, GRA7 and SAG3 vs. UPRT1 and UPRT7 on the other (Supplementary information). A GTR $+\Gamma+$ I model was used with parameters that could vary among data partitions. The different model fits performed with phangorn were compared with AIC. The three trees obtained with the distancebased methods were all tested as initial trees.

\subsection{Geostatistical analyses. We used the same geostatistics approach for inferring} the putative origin of $T$. gondii as previously used for uncovering the origins of $P$. 
falciparum (Tanabe et al., 2010, 2013a, 2013b; Mita and Jombart, 2015). We implemented this approach in the R package geoGraph (http://thibautjombart/geograph), in which we provided extensive documentation replicating the analyses described below using publicly available data (Cann et al., 2002). The method implemented in geoGraph relies on the idea that migration events result in successive bottlenecks which reduce the genetic diversity within populations as they are located further away from the origin (Tanabe et al., 2010). Accordingly, we expect to observe a negative correlation between within-population diversity and the distance from the origin. While in practice the true origin is often unknown, one can infer the most plausible origin by assessing this relationship for a number of candidate origins, and retaining the origin yielding the strongest negative correlation. This method requires two types of distances, genetic and spatial, to be computed. Here, the genetic diversity was mostly structured by varying frequencies of a small number of haplotypes within populations (S3 Table and Fig 1). Therefore, we used haplotype richness (i.e. number of distinct haplotypes) as a measure of diversity within populations. Spatial distances through landmasses were computed using geoGraph. The package models movements on the surface of the Earth using a spherical, pseudo-regular grid with approximately 40,000 nodes. Each node possesses an 'habitat' attribute, here used to distinguish landmasses from seas. Shortest path between locations were computed using the dijkstra algorithm (Jungnickel, 2013) implemented in the R package RBGL (Edmonds et al., 2006; Carey et al., 2011). To 
define candidate origins, 1,800 combinations of regularly spaced longitudes and latitudes were used to cover the globe, which resulted in 433 non-redundant locations on landmasses on the grid used by geoGraph. For each location, the shortest path through each sampled population was identified, and the corresponding distance computed in kilometers. These distances were then used to assess patterns of decrease of genetic diversity from the putative origin using simple linear regression. The most likely origin was inferred as the location which yielded the most negative correlation between geographic distances and haplotype diversity within populations.

2.4. Time to the most recent common ancestor (TMRCA). In order to estimate the time to the most recent common ancestor (TMRCA) of $T$. gondii, we used two different approaches: a simple molecular dating method based on the divergence with $H$. hammondi which is estimated to be around $11 \mathrm{My}$, and a coalescent approach using the expectation of TMRCA which is equal to twice the effective population size ( $\mathrm{Ne})$. Both approaches need an estimate of the mutation rate $(\mu)$, and the second one also needs an estimate of the population parameter $\theta(=2 \mu \mathrm{Ne})$. We estimated $\mu$ for the noncoding introns of UPRT1 and UPRT7. We did two neutrality tests: D's Tajima and the R2 test. We calculated a standard-error of this estimate using the variance of ratio of two random variables, here the number of mutations per site and twice the time of divergence. The former was estimated with a Tamura-Nei distance with its associated 
variance, and the latter was $11 \mathrm{My}$ with an arbitrary sd = $1 \mathrm{My}$. The population parameter $\theta$ was estimated in two ways: with a Markov chain Monte Carlo (MCMC) approach as implemented in the R package coalescentMCMC, and with the nucleotide diversity $(\boldsymbol{\pi})$ calculated with pegas. Both ways calculate the standard-error of the estimate of $\theta$. In the end, three estimates of TMRCA were obtained with their respective 95\% confidence interval $(\mathrm{Cl})$.

\section{Results}

\subsection{Genetic diversity.}

Sequences of the five markers represented a total of 3,153 bp per isolate, including 140 variable sites. Without taking into account sites with gaps, 26, 30, 27, 32, and 25 SNPs were identified in the GRA6 (607 bp), GRA7 (677 bp), SAG3 (638 bp), UPRT1 (574 bp), and UPRT7 (657 bp) genes, respectively (S4 Table and S1 Fig). Strains from the Caribbean, Guiana shield, Northeast Brazil, and Southeast Brazil showed more sequence polymorphism with $65,90,59$, and 65 SNPs, respectively (S3 Table).

Polymorphism was lower in the African and European populations with a number of SNPs ranging from one to 39. Polymorphism was intermediate in the Asian and North American populations with 41, 60, 47, and 51 SNPs in Turkey, China, Minnesota, and Pennsylvania, respectively. 
Data concatenation revealed 60 haplotypes. The number of haplotypes ranged from four in Europe to 32 in South America. Of the 32 genotypes in the 44 strains from South America, 29 were endemic in South America whereas only three were common in other populations (one in the Caribbean, one in Africa, and one both in Asia and Africa). In contrast, of the four genotypes in the 34 strains from Europe, three were common in other populations (one in Asia and Africa, one in North America, Asia and Africa, and one in the Caribbean, North America, Asia and Africa) and the unique genotype differed by only one SNP from the one common to North America, Asia, and Africa (S5 Table). The higher values of haplotype diversity, estimated from concatenation of the five markers by the number of haplotypes divided by the number of isolates, were observed in South America and the Caribbean, whereas the lower values were observed in Europe, Asia, and Africa (S3 Table). Haplotype diversity was intermediate in North America. Overall the highest genetic diversity was found in South-America.

\subsection{Phylogeny of T. gondii strains}

Preliminary analyses of genetic diversity (Supplementary information, S6 Table and S2 and S3 Figs.) revealed different mutation patterns and rates of evolution between coding and non-coding segments, and for the two UPRT genes compared to the others. Accordingly, we defined four partitions of the sequence data crossing these two categories, and reconstructed separate phylogenies by maximum likelihood (ML) to

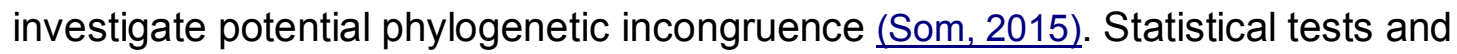


examination of model selection criterion (AIC) revealed the existence of distinct topologies (Fig. 1), suggesting that these sequence partitions have undergone different evolutionary histories and selective pressures. Interestingly, only South American strains were consistently placed at a basal position (close to the root) in all topologies. To investigate this pattern further and identify the common evolutionary history of these genes, a consensus topology was inferred from the four ML-partitioned topologies (Fig. 2). This new tree supported the more ancestral status of South American isolates, with 35 out of 44 samples located at the root of the tree. However, as expected in the presence of conflicting phylogenetic signal, this tree was only partially resolved, and strains from other locations (China: 12 samples; Africa: 7 samples) also belonged to the large basal multifurcation.

\subsection{On the geographic origin of $T$. gondii}

As a complementary analysis, we used a geostatistical approach previously employed for identifying the origin of Plasmodium falciparum, the main etiologic agent of malaria geographic origins as the locations from which patterns of decrease in genetic diversity, expected to be observed due to repeated migration and founder effects, are most consistent (Tanabe et al., 2010). Because of the low level of polymorphism observed in the sequenced genes and the highly clonal nature of $T$. gondii, haplotype richness was used as a measure of genetic diversity within populations (S3 Table). Testing a large number of hypothetical origins across the world, this approach identified South America, and more specifically Colombia as the most likely origin $\left(r=-0.81, p=0.9 \times 10^{-4}\right.$, Fig. 3).

234 While substantial uncertainty remains about the exact location, this analysis brings 
strong support to a South American origin for $T$. gondii suggested by the phylogenetic approach. Our results further suggest that $T$. gondii initially spread through the Americas and then colonized Asia and Europe via the Bering Strait, before entering Africa through two different migration routes (Fig. 3).

\subsection{Time to the most recent common ancestor $\left(T_{M R C A}\right)$ of $T$. gondii.}

To understand the processes which may have led to a pandemic radiation of $T$. gondii from South America, the emergence of this ancestral, highly successful lineage has to be dated. To this end, we derived estimates of time to the most recent common ancestor (MRCA) of the extant strains using standard molecular approaches. While confidence intervals indicated substantial uncertainty, overall results suggest that the MRCA of $T$. gondii appeared around 1.5 My ago (Table 1). This emergence is much more recent that the existence of $T$. gondii itself, estimated to have diverged from its closest ancestor Hammondia hammondi some 11 My ago (Morrison et al., 2004).

\section{Discussion}

A major event occurred in the evolutionary history of $T$. gondii which led to a selective sweep about $1.5 \mathrm{My}$ ago. We hypothesized that an ancestral form of T.gondii was introduced in South America through the migration of Felidae after the emergence of the Isthmus of Panama about 2 to 3 My ago, at the end of Pliocene (O'Brien et al., 2008). It is believed that Felidae species quickly expanded after their arrival and diversified into the "ocelot" lineage in South America. Interestingly the Muridae, potential intermediate hosts for T. gondii, also showed extensive diversification in South America with the 
appearance of several genera (Webb, 2006) around the same time. As previously suggested (Webb, 2006), we posit that this expansion, diversification and mixing of host populations certainly resulted in similar processes in their parasites and favoured the accumulation of genetic diversity in $T$. gondii, which eventually led to a selective sweep by a highly successful mutant lineage.

The selective pressures underlying this selective sweep can be debated. Previous work attributed this radiation to the emergence of transmission through carnivorism (i.e., oral infectivity of tissue cysts) between intermediate hosts in clonal strains 10,000 years ago (Su et al., 2003). However, oral infectivity was shown to be also a trait of many South American strains (Carme et al., 2002; Khan et al., 2007). Because South American strains were the first to diverge from the MRCA, it is likely that transmission by carnivorism evolved earlier than the apparition of clonal lineages. This trait conferred a better transmission of the current form of $T$. gondii which likely outcompeted the ancestral form arriving in South America with Felidae. The transmission of T. gondii between its different hosts would allow some genotypes to migrate to North America, then to go through the Bering Strait to colonize Asia, Europe and Africa. The current population structure of $T$. gondii with a predominance of a few successful clonal strains in Africa, Asia and Europe, is likely to be the consequence of the recent expansion of the domestic cat, an Old World species until the sixteenth century, that tremendously amplified a specific subset of pre-adapted genotypes (Müller and Howard, 2016).

In addition to being the likely origin of modern T. gondii strains, South America also suffers from the highest burden of toxoplasmosis. Prevalence, incidence, and severity of 
acquired and congenital ocular toxoplasmosis (OT) in some areas of Brazil, Colombia, and Argentina are considerably higher than anywhere else, which makes OT a genuine public health issue in South America (Glasner et al., 1992; de-la-Torre et al., 2008; Gilbert et al., 2008; Rudzinski et al., 2016). Because South America is also the hotspot of T. gondii's genetic diversity, it has been hypothesized that severe forms of toxoplasmosis may be the consequence of poor adaptation of the human host to the unusual diversity of strains in this part of the world, resulting in impaired immune response and, thus, a more aggressive disease (Khan et al., 2006; Gilbert et al., 2008; Demar et al., 2012; de-la-Torre et al., 2013; Rudzinski et al., 2016). The societal and economic costs of care for symptomatic cases of congenital toxoplasmosis can be considerable but the cost-effectiveness of national routine prenatal screening and treatment program are still debated (Wallon et al., 1999; Jones et al., 2014). There is a need for randomized placebocontrolled trials to help determine the effectiveness of these interventions.

\section{Conclusion}

Our reconstruction of $T$. gondii's phylogeography provides a new framework for understanding patterns of genetic diversity in sampled populations of the parasite, and for predicting diversity in unsampled locations. Because genetic diversity seems to impact directly the severity of the disease, our results can be used as a basis for explaining geographic heterogeneities in disease burden, and identifying priority targets for potential future interventions. 


\section{ACKNOWLEDGEMENTS}

307 We are thankful to github (http://github.com/) and travis (http://travis-ci.org/) for providing great resources for the development of the R package geoGraph. We are indebted to Endrias Zewdu Gebremedhin, Hüseyin Can, Mert Döşkaya, Yüksel Gürüz, Susana Sousa, Lénaïg Halos, Min Li, François Peyron, and the members of the French National Reference Center for toxoplasmosis (Nicole Desbois, Saadia Azi, Hélène Yera, Magalie Demar, Bernard Carme, Stéphane Simon, Denis Blanchet, Rachida Boukhari, Isabelle Villena, Dominique Aubert) for their contribution to the collection of 168 strains that were included in the analysis.

This research did not receive any specific grant from funding agencies in the public, commercial, or not-for-profit sectors.

The sequences reported in this study have been deposited in the GenBank database (accession n KU598987-KU599154 for GRA6, accession nKU599155-KU599322 for GRA7, accession nKU599323-KU599490 for SAG3, accession nKU599491- 
References

Ajzenberg, D., Daniel, A., Frédéric, C., Aurélien, M., Philippe, V., Marie-Laure, D., 2010. Genotyping of Toxoplasma gondii Isolates with 15 Microsatellite Markers in a Single Multiplex PCR Assay. J. Clin. Microbiol. 48, 4641-4645.

Cann, H.M., de Toma, C., Cazes, L., Legrand, M.-F., Morel, V., Piouffre, L., Bodmer, J., Bodmer, W.F., Bonne-Tamir, B., Cambon-Thomsen, A., Chen, Z., Chu, J., Carcassi, C., Contu, L., Du, R., Excoffier, L., Ferrara, G.B., Friedlaender, J.S., Groot, H., Gurwitz, D., Jenkins, T., Herrera, R.J., Huang, X., Kidd, J., Kidd, K.K., Langaney, A., Lin, A.A., Mehdi, S.Q., Parham, P., Piazza, A., Pistillo, M.P., Qian, Y., Shu, Q., Xu, J., Zhu, S., Weber, J.L., Greely, H.T., Feldman, M.W., Thomas, G., Dausset, J., Cavalli-Sforza, L.L., 2002. A human genome diversity cell line panel. Science 296, 261-262.

Carey, V., Long, L., Gentleman, R., 2011. RBGL: An interface to the BOOST graph library. R package version 1, 124.

Carme, B., Bissuel, F., Ajzenberg, D., Bouyne, R., Aznar, C., Demar, M., Bichat, S., Louvel, D., Bourbigot, A.M., Peneau, C., Neron, P., Darde, M.L., 2002. Severe Acquired

Toxoplasmosis in Immunocompetent Adult Patients in French Guiana. J. Clin. Microbiol. 40, 4037-4044.

de-la-Torre, A., López-Castillo, C.A., Gómez-Marín, J.E., 2008. Incidence and clinical characteristics in a Colombian cohort of ocular toxoplasmosis. Eye 23, 1090-1093.

de-la-Torre, A., Sauer, A., Pfaff, A.W., Bourcier, T., Brunet, J., Speeg-Schatz, C., Ballonzoli, L., Villard, O., Ajzenberg, D., Sundar, N., Grigg, M.E., Gomez-Marin, J.E., Candolfi, E., 2013. Severe South American ocular toxoplasmosis is associated with decreased Ifn-y/ll-17a and increased II-6/II-13 intraocular levels. PLoS Negl. Trop. Dis. 7, e2541.

Demar, M., Hommel, D., Djossou, F., Peneau, C., Boukhari, R., Louvel, D., A.-M., B., Nasser, V., Ajzenberg, D., M.-L., D., Carme, B., 2012. Acute toxoplasmoses in immunocompetent patients hospitalized in an intensive care unit in French Guiana. Clin. Microbiol. Infect. 18, E221-E231.

Edgar, R.C., 2004. MUSCLE: multiple sequence alignment with high accuracy and high throughput. Nucleic Acids Res. 32, 1792-1797.

Edmonds, N., Breuer, A., Gregor, D., Lumsdaine, A., 2006. Single-source shortest paths with the parallel boost graph library. The Ninth DIMACS Implementation Challenge: The Shortest Path Problem, Piscataway, NJ 219-248.

Gilbert, R.E., Freeman, K., Lago, E.G., Bahia-Oliveira, L.M.G., Tan, H.K., Wallon, M., Buffolano, W., Stanford, M.R., Petersen, E., European Multicentre Study on Congenital Toxoplasmosis (EMSCOT), 2008. Ocular sequelae of congenital toxoplasmosis in Brazil compared with Europe. PLoS Negl. Trop. Dis. 2, e277.

Glasner, P.D., Silveira, C., Kruszon-Moran, D., Martins, M.C., Burnier Júnior, M., Silveira, S., Camargo, M.E., Nussenblatt, R.B., Kaslow, R.A., Belfort Júnior, R., 1992. An unusually high prevalence of ocular toxoplasmosis in southern Brazil. Am. J. Ophthalmol. 114, 136- 
144.

Jones, J.L., Parise, M.E., Fiore, A.E., 2014. Neglected parasitic infections in the United States: toxoplasmosis. Am. J. Trop. Med. Hyg. 90, 794-799.

Jungnickel, D., 2013. Graphs, Networks and Algorithms:, Algorithms and Computation in Mathematics. Springer Berlin Heidelberg.

Khan, A., Fux, B., Su, C., Dubey, J.P., Darde, M.L., Ajioka, J.W., Rosenthal, B.M., Sibley, L.D., 2007. Recent transcontinental sweep of Toxoplasma gondii driven by a single monomorphic chromosome. Proc. Natl. Acad. Sci. U. S. A. 104, 14872-14877.

Khan, A., Jordan, C., Muccioli, C., Vallochi, A.L., Rizzo, L.V., Belfort, R., Jr, Vitor, R.W.A., Silveira, C., Sibley, L.D., 2006. Genetic divergence of Toxoplasma gondii strains associated with ocular toxoplasmosis, Brazil. Emerg. Infect. Dis. 12, 942-949.

Lehmann, T., Marcet, P.L., Graham, D.H., Dahl, E.R., Dubey, J.P., 2006. Globalization and the population structure of Toxoplasma gondii. Proc. Natl. Acad. Sci. U. S. A. 103, 1142311428.

Lorenzi, H., Khan, A., Behnke, M.S., Namasivayam, S., Swapna, L.S., Hadjithomas, M., Karamycheva, S., Pinney, D., Brunk, B.P., Ajioka, J.W., Ajzenberg, D., Boothroyd, J.C., Boyle, J.P., Dardé, M.L., Diaz-Miranda, M.A., Dubey, J.P., Fritz, H.M., Gennari, S.M., Gregory, B.D., Kim, K., Saeij, J.P.J., Su, C., White, M.W., Zhu, X.-Q., Howe, D.K., Rosenthal, B.M., Grigg, M.E., Parkinson, J., Liu, L., Kissinger, J.C., Roos, D.S., Sibley, L.D., 2016. Local admixture of amplified and diversified secreted pathogenesis determinants shapes mosaic Toxoplasma gondii genomes. Nat. Commun. 7, 10147.

Mita, T., Jombart, T., 2015. Patterns and dynamics of genetic diversity in Plasmodium falciparum: what past human migrations tell us about malaria. Parasitol. Int. 64, 238-243.

Montoya, J.G., Liesenfeld, O., 2004. Toxoplasmosis. Lancet 363, 1965-1976.

Morrison, D.A., 2005. How old are the extant lineages of Toxoplasma gondii? Parassitologia 47 , 205-214.

Morrison, D.A., Set, B., Per, T., Ulrich, W., Jörg, K., Mattsson, J.G., 2004. The current status of the small subunit rRNA phylogeny of the coccidia (Sporozoa). Int. J. Parasitol. 34, 501-514.

Müller, U.B., Howard, J.C., 2016. The impact of Toxoplasma gondii on the mammalian genome. Curr. Opin. Microbiol. 32, 19-25.

O'Brien, S.J., Johnson, W., Driscoll, C., Pontius, J., Pecon-Slattery, J., Menotti-Raymond, M., 2008. State of cat genomics. Trends Genet. 24, 268-279.

Pappas, G., Roussos, N., Falagas, M.E., 2009. Toxoplasmosis snapshots: global status of Toxoplasma gondii seroprevalence and implications for pregnancy and congenital toxoplasmosis. Int. J. Parasitol. 39, 1385-1394.

Rudzinski, M., Khoury, M., Couto, C., Ajzenberg, D., 2016. Reactivation of Ocular Toxoplasmosis in Non-Hispanic Persons, Misiones Province, Argentina. Emerg. Infect. Dis. $\underline{22,912-913 .}$ 
437

438

439

440

441

442

443

444

445

446

447

448

449

450

451

452

453

454

455

456

457

458

459

460

461

462

463

464

465

466

467

468

469

470

471

472

473

474

475

476

477

478
Shwab, E.K., Zhu, X.-Q., Majumdar, D., Pena, H.F.J., Gennari, S.M., Dubey, J.P., Su, C., 2014. Geographical patterns of Toxoplasma gondii genetic diversity revealed by multilocus PCRRFLP genotyping. Parasitology 141, 453-461.

Som, A., 2015. Causes, consequences and solutions of phylogenetic incongruence. Brief. Bioinform. 16, 536-548.

Su, C., Evans, D., Cole, R.H., Kissinger, J.C., Ajioka, J.W., Sibley, L.D., 2003. Recent Expansion of Toxoplasma Through Enhanced Oral Transmission. Science 299, 414-416.

Tanabe, K., Jombart, T., Horibe, S., Palacpac, N.M.Q., Honma, H., Tachibana, S.-I., Nakamura, M., Horii, T., Kishino, H., Mita, T., 2013a. Plasmodium falciparum mitochondrial genetic diversity exhibits isolation-by-distance patterns supporting a sub-Saharan African origin. Mitochondrion 13, 630-636.

Tanabe, K., Mita, T., Jombart, T., Eriksson, A., Horibe, S., Palacpac, N., Ranford-Cartwright, L., Sawai, H., Sakihama, N., Ohmae, H., Nakamura, M., Ferreira, M.U., Escalante, A.A., Prugnolle, F., Björkman, A., Färnert, A., Kaneko, A., Horii, T., Manica, A., Kishino, H., Balloux, F., 2010. Plasmodium falciparum accompanied the human expansion out of Africa. Curr. Biol. 20, 1283-1289.

Tanabe, K., Mita, T., Palacpac, N.M.Q., Arisue, N., Tougan, T., Kawai, S., Jombart, T., Kobayashi, F., Horii, T., 2013b. Within-population genetic diversity of Plasmodium falciparum vaccine candidate antigens reveals geographic distance from a Central subSaharan African origin. Vaccine 31, 1334-1339.

Torgerson, P.R., Mastroiacovo, P., 2013. The global burden of congenital toxoplasmosis: a systematic review. Bull. World Health Organ 91, 501-508..

Wallon, M., Liou, C., Garner, P., Peyron, F., 1999. Congenital toxoplasmosis: systematic review of evidence of efficacy of treatment in pregnancy. BMJ 318, 1511-1514.

Webb, S.D., 2006. The Great American Biotic Interchange: patterns and processes 1. Ann. Mo. Bot. Gard. 93, 245-257. 
480 Table 1. Estimates of the time to the most recent common ancestor (TMRCA) of 481 Toxoplasma gondii with three different methods on the introns of UPRT genes.

482

\begin{tabular}{lll}
\hline Method & TMRCA $(\mathrm{Ma})$ & {$[95 \% \mathrm{Cl}]$} \\
\hline Molecular dating & 1.59 & {$[0.00-3.46]$} \\
\hline Coalescent & 1.26 & {$[0.94-1.57]$} \\
\hline Nuc. div. $(\boldsymbol{\pi})$ & 1.20 & {$[0.00-2.44]$} \\
\hline
\end{tabular}

483

484 Nuc. div.: nucleotide diversity.

485

486

487

488

489

490

491

492

493

494

495

496

497

498

499

500 